\title{
WEAKENING A THEOREM ON DIVIDED POWERS
}

\author{
BY \\ MOSS E. SWEEDLER( $\left.{ }^{1}\right)$
}

\begin{abstract}
We show that if a Hopf algebra has finite dimensional primitives and a primitive lies in arbitrarily long finite sequences of divided powers then it lies in an infinite sequence of divided powers.
\end{abstract}

Introduction. K. Newman has shown us a counter-example to [3, Theorem 2 , p. 521]. The theorem states that if $H$ is a cocommutative Hopf algebra over a perfect field $k$ of characteristic $p>0$ and $k$ is the unique simple subcoalgebra of $H$ then a primitive element ${ }^{1} x$ of $H$ lies in a sequence of divided powers $1={ }^{0} x,{ }^{1} x, \ldots,{ }^{n+1-1} x$ if and only if ${ }^{1} x$ has coheight $n$, for $n=0,1, \ldots, \infty$. (The sequence should be considered infinite if $n=\infty$.) The proof given in [3, p. 524] correctly shows that the existence of the sequence of divided powers implies that ${ }^{1} x$ has the desired coheight for all $n$. The proof there also correctly shows that for finite $n$ if ${ }^{1} x$ has coheight $n$ then the desired sequence of divided powers exists. The error seems to be the assertion $K^{1} \cong H^{1} /\left(\cup \operatorname{ker} F^{i}\right)$ [3, p. 254, lines 23-24].

Newman's example shows that ${ }^{1} x$ may have infinite coheight-so lies in arbitrarily long finite sequences of divided powers-and yet ${ }^{1} x$ lies in no infinite sequence of divided powers.

We show here that with the further assumption that the primitives of $H$ are finite dimensional then ${ }^{1} x$ having infinite coheight implies that ${ }^{1} x$ lies in an infinite sequence of divided powers. This result is important because it plays a key role in the proof of Jacobson's conjecture in [1]. We explain this in more detail at the end of the present paper.

1. Suppose $C$ is a cocommutative coalgebra with a unique simple subcoalgebra which is one dimensional. We identify this simple subcoalgebra with $k$ and so consider $k \subset C$. Then the primitive elements of $C, P(C) \equiv\{c \in C \mid \Delta c=1 \otimes c+c \otimes 1\}$.

Heyneman's TheOREM. If $\operatorname{dim} P(C)<\infty$ then $C$ satisfies the minimum condition and descending chain condition for subcoalgebras.

Proof. For a vector space $U$ the coalgebra $S h(U)$ is defined in [4, p. 244, p. 254]. By $[4$, p. 254, 12.1.1] there is a finite-dimensional space $U$ and an injective coalgebra

Received by the editors April 7, 1970.

AMS 1969 subject classifications. Primary 1452; Secondary 1450.

Key words and phrases. Coalgebra, cocommutative, coheight, co-Noetherian, irreducible, primitive, sequences of divided powers.

(1) Supported in part by NSF GP-9395.

Copyright (C) 1971, American Mathematical Society 
map $F: C \rightarrow \operatorname{Sh}(U)$. In [4, p. 261] $B(U)$ is defined as the maximal cocommutative subcoalgebra of $\operatorname{Sh}(U)$. Since $C$ is cocommutative $\operatorname{Im} F \subset B(U)$ and we consider $F$ as a coalgebra injection $C \stackrel{F}{\rightarrow} B(U)$. Since $F$ is injective by applying $F$ to the coalgebras in a family of subcoalgebras of $C$ we see that it suffices to show that $B(U)$ has the minimal condition on subcoalgebras. Similarly for the descending chain condition.

By $\left[4\right.$, p. 278 , Example-Exercise] the linear dual $\operatorname{Hom}_{k}(B(U), k)$ is a powerseries ring in $\operatorname{dim} U$ variables; hence, is Noetherian. By [4, p. 16, 1.4.3] if $D$ is a subcoalgebra of $B(U)$ then $D^{\perp}=\left\{f \in \operatorname{Hom}_{k}(B(U), k) \mid f(D)=0\right\}$ is an ideal in $\operatorname{Hom}_{k}(B(U), k)$. Thus the maximal condition and ascending chain condition for ideals in $\mathrm{Hom}_{k}(B(U), k)$ implies the desired conditions for $B(U)$. Q.E.D.

THEOREM 2. Let $k$ be a perfect field of characteristic $p>0$ and $H$ a cocommutative Hopf algebra over $k$ where $k$ is the unique simple subcoalgebra of $H$. Suppose ${ }^{1} x$ is a primitive element of $H$.

(i) If $1={ }^{0} x,{ }^{1} x,{ }^{2} x, \ldots,{ }^{n+1-1} x$ is a sequence of divided powers in $H$ then ${ }^{1} x$ has coheight $n$, for $n=0,1, \ldots, \infty$.

(ii) If ${ }^{1} x$ has coheight $n$ then there is a sequence of divided powers in $H$. $1={ }^{0} x,{ }^{1} x, \ldots,{ }^{p^{n+1}-1} x$, for $n=0,1, \ldots$

(iii) If $\operatorname{dim} P(H)<\infty$ and ${ }^{1} x$ has infinite coheight then there is an infinite sequence of divided powers in $H,\left\{{ }^{i} x\right\}_{i=0}^{\infty}$.

Proof. In [3, p. 524, proof of Theorem 2] statements 1 and 2 are correctly proved. In [3, p. 520, Theorem 1] the $V$ map $V: H \rightarrow H$ is defined. By [3, p. 521, Theorem 1] $V^{n}(V)=V \cdots V(H)$ (n-times) is a sub-Hopf algebra of $H$, thus a subcoalgebra. Assuming $\operatorname{dim} P(H)<\infty$ the descending chain $H \supset V(H) \supset V^{2}(H) \cdots$ must stabilize say at $V^{n}(H)$. Thus $V V^{n}(H)=V^{n}(H)$. Since ${ }^{1} x$ has infinite coheight it follows that ${ }^{1} x \in V^{n}(H)$. Thus $1={ }^{0} x,{ }^{1} x$ may be extended to an infinite sequence of divided powers lying in $V^{n}(H)$ by [3, p. 522, Lemma 7]. Q.E.D.

The statement just above $[1$, p. $285,3.3 .4]$ that $(P H)_{\infty}=\{x \in P H \mid$ there is an infinite sequence of divided powers lying over $x\}$ is false (by Newman's example) unless we have $\operatorname{dim} P H<\infty$, so Theorem 2 (this paper) applies. Thus the proof given for $[1$, p. $285,3.3,4]$ is incorrect unless one assumes that both $P H$ and $P J$ are finite dimensional. The use made of 3.3,4 is [1, p. 290, 3 lines above 3.5] in the proof of 3.5,3, Jacobson's conjecture, where both $P H$ and $P J$ are finite dimensional. (Actually it can be shown by techniques developed by Newman that $3.3,4$ is correct as it stands.)

\section{BIBLIOGRAPHY}

1. H. P. Allen and M. E. Sweedler, A theory of linear descent based upon Hopf algebraic techniques, J. Algebra 12 (1969), 242-294. MR 39 \#4233.

2. R. Heyneman, Coalgebras of finite type (to appear).

3. M. E. Sweedler, Hopf algebras with one grouplike element, Trans. Amer. Math. Soc. 127 (1967), 515-526. MR 35 \#1634.

4. — Hopf algebras, Benjamin, New York, 1969.

CORNELl UNIVERSITY, ITHACA, NEW YORK 14850 